doi:10.13108/2020-12-4-30

\title{
GROWTH ORDER OF SUM OF DIRICHLET SERIES: DEPENDENCE ON COEFFICIENTS AND EXPONENTS
}

\author{
G.A. GAISINA
}

\begin{abstract}
We study the sharpness of the conditions under which the order of the sum of the Dirichlet series converging in some half-plane can be calculated by means of certain formula depending only on the coefficients and exponents. For unbounded functions analytic in the unit circle, a formula of such kind was obtained by a series of scientist in different years, in partucilar, by Govorov in 1959, by MacLane in 1966 and by Sheremeta in 1968. Later an analogue of this notion was also introduced for a Dirichlet series converging in some half-plane. But a corresponding formula for the growth order of the Dirichlet series was established by many authors under strict restrictions. In all previous formulae there were provided the conditions, which were only sufficient for the validity of this formula. In the present work we find conditions being not only sufficient but also necessary for the possibility to calculate the growth order for each Dirichlet series by means of this formula.
\end{abstract}

Keywords: Dirichlet series, half-plane of convergence, formula for the growth order

Mathematics Subject Classification: 30D10

\section{INTRODUCTION}

Entire functions are straightforward generalization of polynomials. If

$$
f(z)=\sum_{n=0}^{\infty} a_{n} z^{n}
$$

is an entire function, by the maximum modulus principle we have

$$
M_{f}(r) \stackrel{\text { def }}{=} \max _{|z|=r}|f(z)|=\max _{|z| \leqslant r}|f(z)| .
$$

Then $M_{f}(r)$ is a non-decreasing on $[0, \infty)$ function and if $f(z) \not \equiv$ const, then $M_{f}(r)$, strictly increasing, tends to $+\infty$ as $r \rightarrow \infty$. For a polynomial $f$ of a degree $n$ we have

$$
\lim _{r \rightarrow \infty} \frac{\ln M_{f}(r)}{\ln r}=n
$$

while for entire transcendental functions the quotient $\frac{\ln M_{f}(r)}{\ln r}$ tends to infinity. This is why the growth $\ln M_{f}(r)$ is compared not with $\ln r$, but with faster growing functions, for instance, with power functions. Proceeding in this way, E. Borel arrived at to the notion of the order $\rho$ of an entire function by letting

$$
\rho=\varlimsup_{r \rightarrow \infty} \frac{\ln \ln M_{f}(r)}{\ln r} .
$$

G.A. Gaisina, Growth order of sum of Dirichlet series: Dependence on Coefficients and EXPONENTS.

(C)Gaisina G.A. 2020.

The research is made in the framework of the development program of Scientific and Educational Mathematical Center of Privolzhsky Federal District, additional agreement no. 075-02-2020-1421/1 to agreement no. 075-02-2020-1421.

Submitted July 11, 2020. 
Comparison of results by J. Hadamard (1893) and E. Borel (1896) showed that the order of entire function (1) is equal to

$$
\rho=\varlimsup_{n \rightarrow \infty} \frac{n \ln n}{\ln \left|1 / a_{n}\right|} .
$$

Let the function $f$ defined by series (1) be analytic only in the circle $D(0,1)=\{z:|z|<1\}$; in this case the convergence radius of series (1) is equal to one. We assume that the function $f$ is unbounded in $D(0,1)$. Then $M_{f}(r) \uparrow \infty$ as $r \uparrow 1$.

An order $\rho$ of an unbounded analytic in the circle $D(0,1)$ function $f$ is the quantity

$$
\rho=\varlimsup_{r \uparrow 1} \frac{\ln \ln M_{f}(r)}{-\ln (1-r)} .
$$

For such functions, the following formula was established independently by N.V. Govorov (1959), G.R. MacLane (1966) and M.N. Sheremerta (1968), see [1]-[3]:

$$
\frac{\rho}{\rho+1}=\varlimsup_{n \rightarrow \infty} \frac{\ln ^{+} \ln ^{+}\left|a_{n}\right|}{\ln n} .
$$

If we let $z=e^{-s}(s=\sigma+i t)$, then we get:

$$
F(s)=f\left(e^{-s}\right)=a_{0}+\sum_{n=1}^{\infty} a_{n} e^{-n s} .
$$

Since under the mentioned change the half-plane $\Pi_{0}^{+}$is mapped onto the unit circle $D(0,1)$, then

$$
M(\sigma) \stackrel{\text { def }}{=} \sup _{|t|<\infty}|F(\sigma+i t)|=M_{f}(r)
$$

where $\sigma>0, r=e^{-\sigma}<1$. It can be confirmed that $-\ln (1-r) \sim-\ln \sigma$ as $r \uparrow 1$; it is also obvious that $\sigma \downarrow 0$. Taking this into consideration, we find:

$$
\rho=\rho_{F} \stackrel{\text { def }}{=} \varlimsup_{\sigma \downarrow 0} \frac{\ln \ln M(\sigma)}{-\ln \sigma} .
$$

Thus, the order $\rho$ of the function $f$ in the circle $D(0,1)$ is equal to the corresponding growth characteristics $\rho_{F}$ of Taylor-Dirichlet series (2). It is called a usual order or simply order of the function $F$ being the sum of series (2). This observation leads us to the notion of a general Dirichlet series

$$
F(s)=\sum_{n=1}^{\infty} a_{n} e^{-\lambda_{n} s}, \quad s=\sigma+i t,
$$

with an arbitrary sequence of exponents $\Lambda=\left\{\lambda_{n}\right\}, 0<\lambda_{n} \uparrow \infty$, converging absolutely (or just uniformly) in some half-plane:

$$
\Pi_{b}=\{s=\sigma+i t: \sigma>b\}, \quad b \in \mathbb{R} .
$$

Thus, there arises a problem on relation of the order of the function $F$ analytic in $\Pi_{b}$ with the coefficients of the expansion of this function into Dirichlet series (3).

Following work [4] by $\mathrm{H}$. Bohr, by $\sigma_{c}, \sigma_{a}, \sigma_{u}$ we denote the abscissas of usual, absolute and uniform convergence of series (3), respectively. As G. Valiron, see [5], [6],

$$
\varlimsup_{n \rightarrow \infty} \frac{\ln \left|a_{n}\right|}{\lambda_{n}} \leqslant \sigma_{c} \leqslant \sigma_{u} \leqslant \sigma_{a} \leqslant \varlimsup_{n \rightarrow \infty} \frac{\ln \left|a_{n}\right|}{\lambda_{n}}+L,
$$

where

$$
L=\varlimsup_{n \rightarrow \infty} \frac{\ln n}{\lambda_{n}}
$$


Generally speaking, in contrast to power series, the quantities $\sigma_{c}, \sigma_{a}, \sigma_{u}$ can be different. As relations (4) show, for $L=0$ they coincide. It can turn out that $\sigma_{u} \neq \sigma_{a}$ and in this case series (3) converges only uniformly and not absolutely. In this case the following formula by M. Kuniyeda holds true [7]:

$$
\sigma_{u}=\varlimsup_{x \rightarrow+\infty} \frac{T(x)}{x}, \quad T(x)=\sup _{|t|<\infty}\left|\sum_{[x] \leqslant \lambda_{n}<x} a_{n} e^{-i \lambda_{n} t}\right|,
$$

where $[x]$ denotes the integer part of a number $x$.

If $\sigma_{u}=-\infty$, then the sum of Dirichlet series (3) is the entire function $F$. In this situation, a most appropriate and convenient characteristics of the growth of the function $F$ is a so-called notion of $R$-order $\rho_{R}$ introduced by J.F. Ritt (1928) [8].

By definition,

$$
\rho_{R}=\varlimsup_{\sigma \rightarrow-\infty} \frac{\ln \ln M_{F}(\sigma)}{-\sigma}
$$

where the quantity $M_{F}(\sigma)$ is defined in the same way as above $q^{1}$. Under the assumption that $\sigma_{a}=-\infty$, i.e., when Dirichlet series (3) converges absolutely in entire plane, J.F. Ritt proved the following formula allowing one to find $\rho_{R}$ (Ritt order) via the coefficients of the expansion:

$$
-\frac{1}{\rho_{R}}=\varlimsup_{n \rightarrow \infty} \frac{\ln \left|a_{n}\right|}{\lambda_{n} \ln \lambda_{n}} .
$$

In work [10] this result was extended for the case of the half-plane $\Pi_{0}$, while in [11] the same was done for a bounded convex domain $G \subset \mathbb{C}$. In the latter case one dealt with series with complex exponents, exponential series, the domain of absolute convergence of which was known to be always convex [9]. In both case there were provided sufficient conditions ensuring the validity of analogues of Ritt formula (6) and depending also on the support function of the convergence domain.

The above described results of works [1]-[3] were generalized for the class $D_{0}(\Lambda)$ of analytic functions represented by Dirichlet series (3) converging absolutely only in the half-plane $\Pi_{0}$. In 1970-1980 this problem was mostly studied by the mathematicians from India, China and Soviet Union. We shall provide a more detailed survey of numerous studies. Now we just say that the matter of these works was to find conditions for the exponents of series (3) ensuring the validity of the formula

$$
\frac{\rho_{F}}{\rho_{F}+1}=\varlimsup_{n \rightarrow \infty} \frac{\ln ^{+} \ln ^{+}\left|a_{n}\right|}{\ln \lambda_{n}}
$$

for the order

$$
\rho_{F}=\varlimsup_{\sigma \rightarrow 0+} \frac{\ln \ln M_{F}(\sigma)}{-\ln \sigma} \quad(\sigma>0) ;
$$

it is assumed that $M_{F}(\sigma) \rightarrow \infty$ as $\sigma \downarrow 0$. These conditions for exponents $\lambda_{n}$ were very different and sometimes too restrictive. At the same time, the sharpness of these conditions was studied in almost in none of works. In paper [12], a weakest condition for $\lambda_{n}$ was nevertheless indicated and it is essentially nature was justified by an example of a particular nature. In a rather recent work [13] made in the Institute of Mathematics of Czech Academy of Sciences in 2012, the result of work [12] was re-proved but in other terms, as we shall see later. Thus, this simple but surely important problem still attracts the attention of some specialists but still remains open. In particular, in the present work we shall prove the necessary part of the theorem from $[12]$.

\footnotetext{
${ }^{1}$ The function $\ln M_{F}(\sigma)$ is convex in the variable $\sigma \in \mathbb{R}[9]$.
} 
In work [14], A.F. Leontiev introduced the notion of order $\rho$ of an analytic function $F$ in a bounded convex domain $G \subset \mathbb{C}$. In the case, when $G$ is a convex polygonal, he proved that each function $F$ analytic in $G$ and obeying in $G$ the estimate

$$
|F(z)| \leqslant e^{\left(\frac{1}{r}\right)^{\rho+\varepsilon}}, \quad r=d(z)=\inf _{\xi \in \partial G}|z-\xi|
$$

$r<r_{0}(\varepsilon), \varepsilon>0$ is arbitrary, can be represented in the domain $G$ by the exponential series

$$
F(z)=\sum_{n=1}^{\infty} a_{n} e^{\lambda_{n} z},
$$

and the series of absolute values satisfies same estimate (8). For $\rho>1$, this result was extended to an arbitrary convex domain by R.S. Yulmukhametov in [15].

However, in works [14], [15], one can not pose the question on validity of some formula like (7) since there is no uniqueness of the expansion into the exponential series and this is why the coefficients of the series are not uniquely defined. This is exactly the main difference between this case and the half-plane.

This problem was solved in work [16], but the estimates for the order $\rho$ obtained in [16] are not sharp. Recently we have obtained sharp two-sided estimates for this order, which will be the matter of another work.

One of the aims in the present paper is to find optimal conditions for the exponents in series (3) ensuring the validity of the formula for the order in the case of the half-plane.

\section{Theorems of Govorov-MacLane type}

1. Case of arbitrary coefficients. We shall assume that $L=0$, this quantity has been defined by formula (5), and $\sigma_{c}=0$. Then Dirichlet series (3) converges absolutely and uniformly in the half-plane $\Pi_{0}$, while its sum $F$ is analytic $\Pi_{0}$. We assume that $M_{F}(\sigma) \rightarrow \infty$ as $\sigma \downarrow 0$, where $M_{F}(\sigma)=\sup _{|t|<\infty}|F(\sigma+i t)|(\sigma>0)$. As above, $D_{0}(\Lambda)$ is the class of analytic in the half-plane $\Pi_{0}$ functions represented by Dirichlet series (3). The quantity

$$
\rho_{F}=\varlimsup_{\sigma \downarrow 0} \frac{\ln \ln M_{F}(\sigma)}{-\ln \sigma}
$$

is called the order of Dirichlet series (3). Exactly in this way this order is defined, for instance, in works [12], [17]-[20]. In [21], [22] the order of function $F \in D_{0}(\Lambda)$ is defined by the formula

$$
\rho_{F}=\varlimsup_{\sigma \downarrow 0} \frac{\ln \ln M_{F}(\sigma)}{-\ln \left(1-e^{-\sigma}\right)},
$$

which obviously coincides with the above introduced order. In above cited papers [18]-[22], the following formula was given with the proof:

$$
\frac{\rho_{F}}{\rho_{F}+1}=\varlimsup_{n \rightarrow \infty} \frac{\ln ^{+} \ln ^{+}\left|a_{n}\right|}{\ln \lambda_{n}},
$$

and it was proved only under additional restrictions for the exponents $\lambda_{n}$ and the coefficients $a_{n}$ of series (3). These conditions were very different and sometimes too restrictive. For instance, it was assumed in [21], [22] that the sequence $\Lambda$ has a finite upper density, that is,

$$
\varlimsup_{n \rightarrow \infty} \frac{n}{\lambda_{n}}=\tau<\infty
$$


As we shall see, this condition is too strong. On the other hand, it was stated in [20] that formula (9) holds under the conditions

$$
\lim _{n \rightarrow \infty} \frac{\ln n}{\lambda_{n}}=\varlimsup_{n \rightarrow \infty} \frac{\ln \left|a_{n}\right|}{\lambda_{n}}=0 .
$$

Here we shall show that only under these conditions, formula (9) is not true, see also [12]. It paper [17], formula (9) was proved but only if

$$
\varlimsup_{n \rightarrow \infty} \frac{\ln n}{\ln \lambda_{n}}=\gamma<\infty .
$$

In the present paper this condition is essentially weakened.

We denote

$$
\alpha=\varlimsup_{n \rightarrow \infty} \frac{\ln \ln n}{\ln \lambda_{n}} .
$$

We also let

$$
\mu=\varlimsup_{n \rightarrow \infty} \frac{\ln ^{+} \ln ^{+}\left|a_{n}\right|}{\ln \lambda_{n}} .
$$

It is stated in paper [18] that if $\alpha \leqslant \mu$, then $\mu=\frac{\rho_{F}}{\rho_{F}+1}$, i.e., formula (9) holds true. A disadvantage of this result is that the condition $\alpha \leqslant \mu$ contains an additional restriction for the coefficients $a_{n}$ in Dirichlet series (3). This is why, according [18], the formula for order $\rho_{F}$ holds not for each function $F$ in the class $D_{0}(\Lambda)$. We shall discuss this issue in Section 2 .

The identity $\mu=\frac{\rho_{F}}{\rho_{F}+1}$ was proved in [12] for $\alpha=0$. And this condition is weaker than condition (10). Indeed, if $\gamma<\infty$, then we obviously have $L=0$ and $\alpha=0$. But there exists a sequence $\Lambda$, for which $\alpha=0, L=0$, but $\gamma=\infty$; it is sufficient to let $\lambda_{n}=e^{\sqrt{n}}, n \geqslant 1$.

In [12], one more fact was established: there exists a sequence $\Lambda$ with $\alpha>0$, there exists a function $F \in D_{0}(\Lambda)$, for which $\mu \neq \frac{\rho_{F}}{\rho_{F}+1}$.

Our aim is to show that condition $\alpha=0$ is in fact necessary. Namely, the following theorem holds true.

Theorem 2.1. For each function $F \in D_{0}(\Lambda)$ the order $\rho_{F}$ is calculated by the formula

$$
\frac{\rho_{F}}{\rho_{F}+1}=\varlimsup_{n \rightarrow \infty} \frac{\ln ^{+} \ln ^{+}\left|a_{n}\right|}{\ln \lambda_{n}}
$$

if and only if $\alpha=0$, that is,

$$
\varlimsup_{n \rightarrow \infty} \frac{\ln \ln n}{\ln \lambda_{n}}=0 .
$$

As a corollary, Theorem 2.1 implies Govorov-MacLane-Sheremeta formula for the order $\rho$ of the function $f$ defined in $D(0,1)$ by series $(1)$.

We note that in work [13] published in 2012, the identity

$$
\mu=\frac{\rho_{F}}{\rho_{F}+1}
$$

was proved under the assumption $\alpha_{0}=0$, where

$$
\alpha_{0}=\varlimsup_{k \rightarrow \infty} \frac{\ln ^{+} \ln \left(p_{k}+1\right)}{\ln k}=0
$$

where $p_{k}+1$ is the number of the points $\lambda_{n}$ in the half-interval $[k, k+1)$. But this statement is in fact the sufficient part of the theorem proved completely in [12] by A.M. Gaisin in 1981. 
Let us show that $\alpha_{0}=0$ if and only if $\alpha=0$. Indeed, if $\alpha=0$ then it is obvious that $\alpha_{0}=0$. Let $\lambda_{j}$ be a point closest from the left to $(k+1)$. Then

$$
\frac{\ln \ln \left(p_{k}+1\right)}{\ln k} \leqslant \frac{\ln \ln j}{\ln k} \leqslant \frac{\ln (k+1)}{\ln k} \frac{\ln \ln j}{\ln \lambda_{j}} \rightarrow 0
$$

as $j \rightarrow \infty$. Hence, $\alpha_{0}=0$.

Let $\alpha_{0}=0$. Then for each $\varepsilon>0$, as $i>i_{0}(\varepsilon)$ we have:

$$
p_{i}+1<e^{i^{\varepsilon}} .
$$

Let $\lambda_{n} \in[k, k+1)$. Then, as $i>i_{1}(\varepsilon)>i_{0}(\varepsilon)$,

$$
n \leqslant n\left(i_{0}\right)+\sum_{i=i_{0}+1}^{k} e^{i^{\varepsilon}} \leqslant n\left(i_{0}\right)+e^{k^{\varepsilon}} k<2 \lambda_{n} e^{\lambda_{n}^{\varepsilon}} .
$$

This shows that $L=0$. Hence, $\sigma_{c}=\sigma_{a}=\sigma_{u}=0$. Moreover, as $n>n_{0}(\varepsilon)$,

$$
\ln n<2 \lambda_{n}^{\varepsilon} \text {. }
$$

Therefore,

$$
\varlimsup_{n \rightarrow \infty} \frac{\ln \ln n}{\ln \lambda_{n}} \leqslant \varepsilon .
$$

Since $\varepsilon>0$ is arbitrary, we conclude that $\alpha=0$.

Proof of Theorem 2.1. . The sufficiency was established in [12]. At that, formula holds also for the case $\rho_{F}=\infty$. Then it remains to prove the necessity only.

Let, as above,

$$
\alpha=\varlimsup_{n \rightarrow \infty} \frac{\ln \ln n}{\ln \lambda_{n}} .
$$

This means that for each $\beta, 0<\beta<\alpha$, there exists a sequence $\left\{n_{m}\right\}$ of natural numbers $n_{m}$, $n_{m} \uparrow \infty$, such that

$$
\frac{\ln \ln n_{m}}{\ln \lambda_{n_{m}}} \geqslant \beta>0 .
$$

Since by assumptions, $L=0$, it is easy to confirm that $\alpha \leqslant 1$. Then $\beta<1$.

We consider the series

$$
F(s)=\sum_{n=1}^{\infty} a_{n} e^{-\lambda_{n} s} \quad(s=\sigma+i t),
$$

where $a_{n}=e$. As above, we assume that the condition holds: $\ln n=o\left(\lambda_{n}\right)$ as $n \rightarrow \infty$. Series (13) converges and by the latter condition, it also converges absolutely in the right half-plane $\Pi_{0}$. Calculating the order by formula (11), we have: $\rho_{F}=0$. We are going to make sure that the order satisfies the inequality $\rho_{F}>0$. This will imply that the sum of series (13) is unbounded in $\Pi_{0}$, that is, $F \in D_{0}(\Lambda)$.

Indeed, since $a_{n}>0$, then

$$
M_{F}(\sigma)=\sup _{|t|<\infty}|F(\sigma+i t)| \geqslant|F(\sigma)|=e \sum_{n=1}^{\infty} e^{-\lambda_{n} \sigma} \quad(\sigma>0) .
$$

On the other hand, it is obvious that

$$
M_{F}(\sigma) \leqslant e \sum_{n=1}^{\infty} e^{-\lambda_{n} \sigma} .
$$


Therefore, $M_{F}(\sigma)=|F(\sigma)|$. Employing estimate $M_{F}(\sigma) \geqslant|F(\sigma)|$, for each natural $N$ we have:

$$
M_{F}(\sigma) \geqslant e \sum_{n=\left[\frac{N}{2}\right]}^{N} e^{-\lambda_{n} \sigma} \geqslant e \frac{N}{2} e^{-\lambda_{N} \sigma} \geqslant N e^{-\lambda_{N} \sigma},
$$

where $[a]$ is the integer part of $a$. Taking into consideration $(12)$, we let $N=n_{m}(m=1,2, \ldots)$. Then we obtain:

$$
M_{F}(\sigma) \geqslant n_{m} e^{-\lambda_{n_{m}} \sigma}=\exp \left[\ln n_{m}-\lambda_{n_{m}} \sigma\right] \quad(\sigma>0) .
$$

Now it follows from relation (12) that

$$
\lambda_{n_{m}} \leqslant\left(\ln n_{m}\right)^{\frac{1}{\beta}} \quad(m \geqslant 1) .
$$

Hence, we get:

$$
M_{F}(\sigma) \geqslant \exp \left[\ln n_{m}-\left(\ln n_{m}\right)^{\frac{1}{\beta}} \sigma\right] \quad(m \geqslant 1),
$$

where $0<\beta<\alpha \leqslant 1$ and $\sigma>0$ is arbitrary.

Since $\beta<1$, as $\sigma$ we can take a solution $\sigma_{m}$ to the equation

$$
\ln n_{m}=2\left(\ln n_{m}\right)^{\frac{1}{\beta}} \sigma,
$$

or, which is the same,

$$
2\left(\ln n_{m}\right)^{\frac{1}{\beta}-1}=\frac{1}{\sigma} \quad(0<\beta<1) .
$$

Then, taking into consideration (15), by (14) we obtain:

$$
\ln M_{F}\left(\sigma_{m}\right) \geqslant\left(\ln n_{m}\right)^{\frac{1}{\beta}}\left[\left(\ln n_{m}\right)^{1-\frac{1}{\beta}}-\sigma_{m}\right]=\left(\ln n_{m}\right)^{\frac{1}{\beta}} \sigma_{m}=\left(\frac{1}{2}\right)^{\frac{1}{1-\beta}}\left(\frac{1}{\sigma_{m}}\right)^{\frac{\beta}{1-\beta}} \quad(m \geqslant 1) .
$$

Hence, we obtain that as $m \rightarrow \infty$

$$
\ln M_{F}\left(\sigma_{m}\right) \geqslant\left(\frac{1}{2}\right)^{\frac{1}{1-\beta}}\left(\frac{1}{\sigma_{m}}\right)^{\frac{\beta}{1-\beta}} .
$$

This finally implies:

$$
\ln \ln M_{F}\left(\sigma_{m}\right) \geqslant[1+o(1)] \frac{\beta}{1-\beta} \ln \frac{1}{\sigma_{m}}, \quad m \rightarrow \infty .
$$

Since $\sigma_{m} \rightarrow 0$ as $m \rightarrow \infty$, then

$$
\rho_{F}=\varlimsup_{\sigma \downarrow 0} \frac{\ln \ln M_{F}(\sigma)}{-\ln \sigma} \geqslant \frac{\beta}{1-\beta} \quad(0<\beta<\alpha \leqslant 1) .
$$

Since $\beta<\alpha$ is an arbitrary positive number, we see that if $\alpha=1$, then $\rho_{F}=\infty$. As $\alpha<1$ we have:

$$
\rho_{F} \geqslant \frac{\alpha}{1-\alpha}>0, \quad 0<\alpha<1
$$

To provide a full arguing, let us show that the order $\rho_{F}$ is equal to $\frac{\alpha}{1-\alpha}$. Indeed, for each $\varepsilon>0$

$$
\frac{\ln \ln n}{\ln \lambda_{n}}<q, \quad q=\alpha+\varepsilon,
$$

as $n \geqslant n_{0}(\varepsilon)$. Since $\alpha<1$, by an appropriate choice $\varepsilon>0$ we can suppose that $q<1$.

Thus,

$$
M_{F}(\sigma) \leqslant e \sum_{n=1}^{\infty} \frac{1}{n^{2}} \exp \left[\left(2 \ln n-\lambda_{n} \sigma\right)\right] \leqslant \frac{\pi^{2} e}{6} \exp \left[\max _{n \geqslant 1}\left(2 \ln n-\lambda_{n} \sigma\right)\right] \quad(\sigma>0) .
$$


Taking into consideration (17), (18), we obtain:

$$
M_{F}(\sigma) \leqslant \frac{\pi^{2} e}{6} \exp \left[\max _{x \geqslant 0}\left(2 x-x^{\frac{1}{q}} \sigma\right)\right] .
$$

The maximum is attained at the point $x_{0}$ not exceeding $x_{1}$, where $x_{1}$ is a root of the equation $2 x=x^{\frac{1}{q}} \sigma$, that is, $2 x^{\frac{q-1}{q}}=\sigma$. This yields

$$
x_{1}=2^{\frac{q}{1-q}}\left(\frac{1}{\sigma}\right)^{\frac{q}{1-q}} .
$$

Hence,

$$
M_{F}(\sigma) \leqslant \frac{\pi^{2} e}{6} e^{2 x_{1}}=\frac{\pi^{2} e}{6} \exp \left[2^{\frac{q}{1-q}}\left(\frac{1}{\sigma}\right)^{\frac{q}{1-q}}\right] .
$$

Therefore, since $\ln (1+x) \sim x$ as $x \rightarrow 0$, we find:

$$
\frac{\ln \ln M_{F}(\sigma)}{-\ln \sigma} \leqslant[1+o(1)] \frac{q}{1-q} \text {. }
$$

This means that $\rho_{F} \leqslant \frac{q}{1-q}, q=\alpha+\varepsilon$. But $\varepsilon>0$ is arbitrary and therefore, $\rho_{F} \leqslant \frac{\alpha}{1-\alpha}$. Now by the inverse inequality we conclude that $\rho_{F}=\frac{\alpha}{1-\alpha}$. Since we obviously have $F \in D_{0}(\Lambda)$, the necessity of the theorem is completely proved.

2. Case of consistent exponents and coefficients. Here we are going to prove a similar theorem under some consistency condition for $\lambda_{n}$ and the coefficients $a_{n}$ of the Dirichlet series.

Let, as above,

$$
\alpha=\varlimsup_{n \rightarrow \infty} \frac{\ln \ln n}{\ln \lambda_{n}}
$$

It was shown that $0 \leqslant \alpha \leqslant 1$. We denote

$$
\mu=\varlimsup_{n \rightarrow \infty} \frac{\ln ^{+} \ln ^{+}\left|a_{n}\right|}{\ln \lambda_{n}} .
$$

It was stated in paper [18] that if $\alpha$ is fixed and the parameter $\mu$ satisfies the inequality $\mu \geqslant \alpha$, then $\mu=\frac{\rho_{F}}{\rho_{F}+1}$, that is, formula (11) holds. A complete proof of this statement was provided in [23]. A disadvantage of this results is that the condition $\alpha \leqslant \mu$ for a fixed $\Lambda$ involves an additional restrictions for the coefficients $a_{n}$ of Dirichlet series (3). This is why a formula for the order $\rho_{F}$ can not hold for any function $F$ in the class $D_{0}(\Lambda)$. Therefore, it is natural to pose a question how the condition $\mu \geqslant \alpha$ is essential for the validity of formula (11). Below we shall provide an answer for this question.

Let $\mu(0 \leqslant \mu \leqslant 1), \alpha(0 \leqslant \alpha \leqslant 1)$ be given numbers. By $D_{0}(\mu, \alpha)$ we denote a subclass of the class $D_{0}(\Lambda)$ of Dirichlet series (3), the coefficients $a_{n}$ of which satisfy inequality (20), while the exponents $\lambda_{n}$ obey identity (19). As it has been said above (see [23]), as $\alpha \leqslant \mu$, the order $\rho_{F}$ of each function $F \in D_{0}(\mu, \alpha)$ can be calculated by means of formula (11).

We note that the restrictions $0 \leqslant \alpha \leqslant 1$ and $0 \leqslant \mu \leqslant 1$ are implied by identities (19), (20).

For $\mu=1$ formula (11) holds since in this case $\alpha \leqslant \mu$. At that, $\mu=\frac{\rho_{F}}{\rho_{F}+1}=1$, that is, $\rho_{F}=\infty$. This is why in what follows we assume that $\mu<1$. We shall show that for arbitrary numbers $\alpha(0 \leqslant \alpha \leqslant 1), \mu(0 \leqslant \mu<1)$ such that $\mu<\alpha$, there exists a function $F \in D_{0}(\mu, \alpha)$, for which the order $\rho_{F}$ can not be found by formula (11). This means that the following theorem holds true.

Theorem 2.2. Assume that numbers $\mu(0 \leqslant \mu<1), \alpha(0 \leqslant \alpha \leqslant 1)$ are given. The order $\rho_{F}$ of each function $F \in D_{0}(\mu, \alpha)$ is calculated by formula (11) if and only if $\alpha \leqslant \mu$. 
Proof. The sufficiency of this theorem was proved in [23]. Let us prove the necessity of the condition $\alpha \leqslant \mu$. Let $\mu<\alpha$. This means for each $\varepsilon>0$ there exists a sequence $\left\{n_{m}\right\}$ of natural numbers $n_{m}, n_{m} \uparrow \infty$, such that

$$
\beta_{1} \leqslant \frac{\ln \ln n_{m}}{\ln \lambda_{n_{m}}} \leqslant \beta_{2} \quad(m \geqslant 1),
$$

where $\beta_{1}=\alpha-\varepsilon, \beta_{2}=\alpha+\varepsilon(0<\varepsilon<\alpha)$.

Fixing the sequence $\left\{n_{m}\right\}$, we are going to construct an appropriate example of the function $F \in D_{0}(\mu, \alpha)$. In order to do this, we choose the coefficients $a_{n}$ and exponents $\lambda_{n}$ of Dirichlet series (3) in a special way but satisfying respectively conditions (19) and (20).

We let

$$
a_{n}=\exp \left[\left(\ln n_{m}\right)^{\frac{\mu}{\alpha}}\right] \quad(0 \leqslant \mu \leqslant \alpha), \quad n_{m} \leqslant n<n_{m+1} \quad(m \geqslant 1) ;
$$

we shall choose the exponents $\lambda_{n}$ of series (3) later. It is clear, for this series with such coefficients the convergence domain and the domain of the absolute convergence is $\Pi_{0}[24]$. For such coefficients $a_{n}$, we let

$$
\nu=\varlimsup_{n \rightarrow \infty} \frac{\ln ^{+} \ln ^{+}\left|a_{n}\right|}{\ln \lambda_{n}}
$$

and we are going to confirm that $\nu=\mu$. Indeed, let $n_{m} \leqslant n<n_{m+1}$. Then

$$
\frac{\ln \ln \left|a_{n}\right|}{\ln \lambda_{n}}=\frac{\mu}{\alpha} \frac{\ln \ln n_{m}}{\ln \lambda_{n}} \leqslant \frac{\mu}{\alpha} \frac{\ln \ln n_{m}}{\ln \lambda_{n_{m}}} .
$$

Taking into consideration (21), we then get that $\nu \leqslant \frac{\mu}{\alpha}(\alpha+\varepsilon)$. Since $\varepsilon>0$ is arbitrary, then $\nu \leqslant \mu$. On the other hand,

$$
\nu \geqslant \varlimsup_{m \rightarrow \infty} \frac{\ln ^{+} \ln ^{+}\left|a_{n}\right|}{\ln \lambda_{n_{m}}}=\frac{\mu}{\alpha} \varlimsup_{m \rightarrow \infty} \frac{\ln \ln n_{m}}{\ln \lambda_{n_{m}}} .
$$

Therefore, taking into consideration the left estimate in (21), we obtain that $\nu \geqslant \frac{\mu}{\alpha}(\alpha-\varepsilon)$, i.e., $\nu \geqslant \mu$. Thus, $\nu=\mu$.

If the order $\rho_{F}$ of the Dirichlet series

$$
F(s)=\sum_{n=1}^{\infty} a_{n} e^{-\lambda_{n} s} \quad(s=\sigma+i t, \sigma>0)
$$

had been calculated by formula (11), we would have

$$
\rho_{F}=\frac{\mu}{1-\mu} .
$$

Let us make sure that under an appropriate choice of the exponents of series (22) this is not true. Indeed, since $a_{n}>0$, we have:

$$
M_{F}(\sigma)=\sup _{|t|<\infty}|F(\sigma+i t)| \geqslant|F(\sigma)|=\sum_{n=1}^{\infty} a_{n} e^{-\lambda_{n} \sigma} \quad(\sigma>0) .
$$

Therefore,

$$
M_{F}(\sigma) \geqslant \sum_{n=n_{m}}^{2 n_{m}} a_{n} e^{-\lambda_{n} \sigma} \geqslant n_{m} \exp \left[\left(\ln n_{m}\right)^{\frac{\mu}{\alpha}}-\lambda_{2 n_{m}} \sigma\right] .
$$

We let $\lambda_{n}=(\ln n)^{\frac{1}{\alpha}}(n \geqslant 2)$. Then the sequence $\Lambda=\left\{\lambda_{n}\right\}$ satisfies condition (19), and $\lambda_{2 n_{m}}=\left(\ln 2 n_{m}\right)^{\frac{1}{\alpha}}$. Thus, by (24) we get

$$
\ln M_{F}(\sigma) \geqslant \ln n_{m}+\left(\ln n_{m}\right)^{\frac{\mu}{\alpha}}-\left(\ln 2 n_{m}\right)^{\frac{1}{\alpha}} \sigma \quad(\sigma>0) .
$$


We choose $\sigma=\sigma_{m}$ as a solution to the equation

$$
\left(\ln n_{m}\right)^{\frac{\mu}{\alpha}}=\left(\ln 2 n_{m}\right)^{\frac{1}{\alpha}} \sigma .
$$

Since $\left(\ln 2 n_{m}\right)^{\frac{1}{\alpha}}=(1+o(1))\left(\ln n_{m}\right)^{\frac{1}{\alpha}}$ as $m \rightarrow \infty$, it follows from $(26)$ that as $m \rightarrow \infty$, the identity holds:

$$
\frac{1}{\sigma}=(1+o(1))\left(\ln n_{m}\right)^{\frac{1-\mu}{\alpha}} .
$$

Hence, taking into consideration (26), (27), by (25) we obtain that, as $m \rightarrow \infty$,

$$
\ln M_{F}\left(\sigma_{m}\right) \geqslant \ln n_{m}=(1+o(1))\left(\frac{1}{\sigma}\right)^{\frac{\alpha}{1-\mu}} \text {. }
$$

This means that $\rho_{F} \geqslant \frac{\alpha}{1-\mu}$. But, according (23), $\rho_{F}=\frac{\mu}{1-\mu}$. Hence, in view of the previous estimate,

$$
\frac{\mu}{1-\mu} \geqslant \frac{\alpha}{1-\mu} \quad(0 \leqslant \mu<1)
$$

which contradicts the assumption $\mu<\alpha$. The proof is complete.

Let $D(0,1)$ be the convergence circle of power series (1). For the Taylor-Dirichlet series

$$
\sum_{n=1}^{\infty} a_{n} e^{-n s}
$$

the usual order $\rho_{F}$ coincides with the order $\rho$ of the function $f$ of form (1). Since in this case $\lambda_{n}=n$, then

$$
\alpha=\lim _{n \rightarrow \infty} \frac{\ln \ln n}{\ln \lambda_{n}}=0,
$$

and Theorem 2.1 implies the aforementioned Govorov-MacLane-Sheremeta formula for calculating the order $\rho$ of the function $f$ defined in the circle $D(0,1)$ by series $(1)$.

\section{BIBLIOGRAPHY}

1. N.V. Govorov. On relation between growth of the function analytic in a circle and coefficients of its power expansion // Trudy Novocherkassk. Politekhn. Inst. 100, 101-115 (1959). (in Russian).

2. G.R. MacLane. Asymptotic values of holomorphic functions. Rice Univ., Houston (1963).

3. M.N. Sheremeta. The connection between the growth of functions of order zero which are entire or analytic in a disc and their power series coefficients // Izv. VUZov. Matem. 6, 115-121 (1968). (in Russian).

4. H. Bohr. Collected Mathematical Works. Dansk Matematisk Forening, Copenhagen (1952).

5. G. Valiron. Sur l'abscisse de convergence des séries de Dirichlet // S. M. F. Bull. 52, 166-174 (1924).

6. G. Valiron. Entire functions and Borel's directions // Proc. Natl. Acad. Sci. USA. 20:3, 211-215 (1934).

7. M. Kuniyeda. Uniform convergence-abscissa of general Dirichlet series // Tohoku Math. J. 9, 7-27 (1916).

8. J.F. Ritt. On certain points in the theory of Dirichlet series // Amer. J. Math. 50:1, 73-86 (1928).

9. A.F. Leontiev. Exponential series. Nauka, Moscow (1976). (in Russian).

10. A.M. Gaussin. A bound for the growth in a half-strip of a function represented by a Dirichlet series // Matem. Sbornik. 117(159):3, 412-424 (1982). [Math. USSR-Sbornik. 43:3, 411-422 (1983).]

11. A.M. Gaisin. Behavior of the sum of a series of exponentials near the boundary of the domain of regularity // Matem. Zametki. 48:3, 45-53 (1990). [Math. Notes. 48:3, 904-910 (1990).] 
12. A.M. Gaisin. On growth of function represented by Dirichlet series near convergence line // in "Studies on approximation theory of functions". Bashkir Branch of AS of USSR, 5-13 (1981). (in Russian).

13. Zhendog Gu, Daochun Sun. The growth of Dirichlet series // Czechoslovak Math. J. 62:1, 29-38 (2012).

14. A.F. Leont'ev. Exponential series for functions with specified growth near the boundary // Izv. AN SSSR. Ser. Matem. 44:6, 1308-1328 (1980). [Math. USSR-Izv. 17:3, 505-521 (1981).]

15. R.S. Yulmukhametov. Space of analytic functions with prescribed growth near the boundary // Matem. Zametki. 32:1, 41-57 (1982). [Math. Notes. 32:1, 499-508 (1982).]

16. A.M. Gaisin, G.A. Gaisina. Behavior of coefficients of series of exponents of finite order near the boundary // Itogi Nauki i Tekhniki. Ser. Sovrem. Mat. Pril. Temat. Obz. 162, 15-24 (2019). (in Russian).

17. E.Ya. Dagene. On central exponent of Dirichlet series // Litov. Matem. Sborn. 8:3, 504-521 (1968). (in Russian).

18. V.S. Boichuk. On growth of Dirichlet series absolutely converging in half-plane // Matem. Sborn. (Naukova Dumka, Kiev), 238-240 (1976).

19. Yu. M. Gal', M.N. Seremeta. On the growth of functions analytical in a half-plane and given by Dirichlet series // Dopov. Akad. Nauk Ukr. RSR, Ser. A. 12, 1064-1067 (1978). (in Ukrainian).

20. Yu-Chia-Yung. Sur la croissance et la repartition de Dirichlet qui ne convergent que dans un demi-plan // C.R. Acad. Sci. Ser A. 288:19, 891-893 (1979).

21. K. Nandan. On the maximum terms and maximum modulus analytic functions represented by Dirichlet series // Ann. Polon. Math. 28, 213-222 (1973).

22. K. Nandan. On the lower order of analytic functions represented by Dirichlet series // Rev. roum. math. pures et appl. 21:10, 1361-1368 (1976).

23. A.M. Gaisin. Behavior of sum of Dirichlet series near the boundary of regularity domain. PhD thesis, Ufa (1982). (in Russian).

24. A.F. Leontiev. Entire functions. Exponential series. Nauka, Moscow (1983). (in Russian).

Galia Akhtyarovna Gaisina,

Bashkir State University,

Zaki Validi str. 32,

450076, Ufa, Russia

E-mail: gaisinaga@mail.ru 